\title{
Dyslipidemia, Diabetes Mellitus, Rheumatoid Arthritis: Association Study Between them in a Group of Individuals in Shijak Population
}

\author{
Brisida Shera \\ Department of Biology, Faculty of Natural Sciences, Tirana University, Albania
}

\begin{abstract}
The aim of the study was to evaluate the association between Dyslipidemia, Diabetes Mellitus and Rheumatoid Arthritis in a population of 289 individuals ( 154 female and 135 male ), for the year 2020 ( January - December ) and the year 2021 ( January - July ), where $85.8 \%$ are patients with Diabetes Mellitus ( DM ) , $9.7 \%$ are patients with Dyslipidemia and $4.5 \%$ are patients with Rheumatoid Arthritis ( RA ). Diseases are analyzed separately from each - other calculating the correlation based on the values of the main analytical components for each disease. From this study it was determined that the number of individuals with DM and Dyslipidemia is the highest in the age group VII ( 65 years and above ). The highest average value of glycemia is in the age group V ( $45-54$ years old ), with value $195 \mathrm{mg} / \mathrm{dl}$ for the female gender and $218.4 \mathrm{mg} / \mathrm{dl}$ for the male gender. In individuals with DM has significant correlation values between glycemic - triglycerides with Pearson correlation Value 0.157. Individuals with Diabetes have a high tendency to be affected by Dyslipidemia. In patients with Dyslipidemia significant correlation value are between Cholesterol- Glycemi with Pearson correlation value 0. 555. Individuals with Dyslipidemia have a high tendency to be affected by DM. In patients with RA the largest number of individuals is in the age group V and VI. Females are in greater number than males. The highest erythrocyte sedimentation rates are in the age group VII, with average value $21.5 \mathrm{mg} / \mathrm{dl}$. Higher correlation value is between erythrosediment - LDL- Ch with value 0.93 . Individuals with RA have a high tendency to be affected by Dyslipidemia. The knowledge gained from this study are important to determine the correct diagnosis for primary and concomitant diseases recognizing risk factors. Prevention of diseases at an early stage and prevention of comorbidities to avoid deteriorating health
\end{abstract}

Keywords: Erythrosediment, Diabetes Mellitus, Dyslipidemia, Rheumatoid Arthritis, Triglyeride

\section{Introduction}

Dyslipidemia: is, quite simply "abnormal lipid levels", as measured on a blood sample and which reflects one of several disorders in the metabolism of lipoproteins. Risk factors for Dyslipidemia: Men who are 40 years of age or more. Women who are postmenopausal or over 50 years of age. Familial hypercholesterolemia, diabetes, hypertension, smoking, Abdominal obesity, strong family history of premature cardiovascular disease (CVD), manifestations of hypercholesterolemia, evidence of symptomatic or asymptomatic atherosclerosis ( Building Healthy lifestyles, Clinical Guide 2006 ). Dyslipidemia may have pathophysiological components that are genetic, environ-mental, or both. Genetic errors of cholesterol synthesis regulation, hepatic cholesterol metabolism, cell membrane receptor function, and others are recognized, yet poorly understood. Lifestyle factors including dietary habits and activity levels are also well recognized, and their modification often constitutes initial conservative interventions in the treatment of dyslipidemia. Risk for dyslipidemia is increased by a variety of factors, including family history, aging, weight gain, physical inactivity, menopause, insulin-resistance, diseases such as type 2 diabetes mellitus and hypothyroidism, and diets high in saturated fats, trans fats, and cholesterol. Cigarette smoking is another risk factor that contributes to low HDL cholesterol ( Jim Gerber ). Dyslipidemia can be classified as hypercholesterolemia, mixed (combined) dyslipidemia, hypertriglyceridemia, and severe hypertriglyceridemia ( MOH ( Ministry of Health Singapore Clinical Practice Guidelines ) 2016 ). 
Nowadays, cardiovascular disease is considered as a leading cause of mortality worldwide, and dyslipidemia is one of its most common risk factors (Abdalrashid Faisal M Halawani et al., 2019). Diabetes is a group of metabolic diseases characterized by hyperglycemia resulting from defects in insulin secretion, insulin action, or both. Several pathogenic processes are involved in the development of diabetes. The basis of the abnormalities in carbohydrate, fat, and protein metabolism in diabetes is deficient action of insulin on target tissues. Deficient insulin action results from inadequate insulin secretion and diminished tissue responses to insulin at one or more points in the complex pathways of hormone action (American Diabetes Association 2014 ). Diabetes is a highly disabling disease, which can cause blindness, amputations, kidney disease, anemia, and cardiovascular and brain complications, among others, impairing the functional capacity and autonomy and individual quality of life ( Jessica Barbieri et al., 2015 ). Diabetic dyslipidemia is characterized by elevated serum triglyceride (TG), low density lipoprotein cholesterol (LDL-C) and decreased serum high density lipoprotein cholesterol (HDL-C) levels. The underlying pathophysiology is complex and is partially understood. Overproduction of very low density lipoprotein cholesterol (VLDL-C) by liver, elevated free fatty acid levels, and disrupted activity of lipoprotein lipase are some of the underlying mechanisms. These changes in the lipid levels are associated with increased cardiovascular disease (CVD) risk in diabetic patients. Diabetic dyslipidemia should absolutely be treated, and life style modification, diet, and medical treatments are main treatments of dyslipidemia ( Yasin Simsek, Halit Diri, Fahri Bayram 2015 Tip 2 Diabetes Mellitus ve Dislipidemi ). Type 2 diabetes is associated with chronic, low-grade inflammation and could potentially trigger the progression of other, more prominent inflammatory diseases such as rheumatoid arthritis (RA) ( Ming-Chi Lu 2014 ). Rheumatoid arthritis is the most commonly diagnosed systemic inflammatory arthritis. Women, smokers, and those with a family history of the disease are most often affected. Criteria for diagnosis include having at least one joint with definite swelling that is not explained by another disease. In a patient with inflammatory arthritis, the presence of a rheumatoid factor or anti-citrullinated protein antibody, or elevated C-reactive protein level or erythrocyte sedimentation rate suggests a diagnosis of rheumatoid arthritis. Older age, a family history of the disease, and female sex are associated with increased risk of RA, although the sex differential is less prominent in older patients. Both current and prior cigarette smoking increases the risk of RA (American Family Physician 2011 ). There is now a large body of epidemiological evidence linking rheumatoid arthritis (RA) with the premature development of cardiovascular disease. This relates, at least in part, to the systemic inflammatory burden in RA, which has been shown to predispose to the development of premature atherosclerosis in individuals with this condition. Raised levels of systemic inflammation have also been shown to predispose to developing both insulin resistance and type 2 diabetes mellitus. ( The Journal of Rheumatology 2007 ). Cardiovascular disease in RA may result from accelerated atherosclerosis caused by clinical or subclinical vasculitis. The main determinants of cardiovascular risk are concentrations of serum low density lipoproteins (LDL), high density lipoproteins (HDL) and triglycerides (Shelja Deswal et al., 2015 ).

\section{Materials and Methods}

Analysis data were obtained from the laboratory of Shijak State Hospital, Durrës, Albania, in the time period January - December 2020 and January - July 2021.

Data on the geographical position of Shijak:

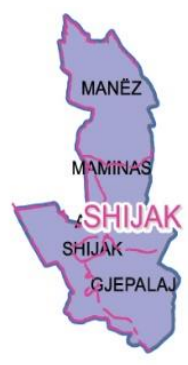

Figure 1: Map of Shijak Municipality 
Shijak municipality is located in the part of central Albania and is part of administrative district of Durres and is bordered by Xhafzotaj, Gjepalaj and Maminas municipalities. In its composition there are respectively 3 neighborhoods: 1. Popular neighborhood; 2. Erzen neighborhood; 3. Kodra neighborhood ( Wikipedia, Shijaku ).

For the Dyslipidemia, Diabetes Mellitus and Rheumatoid Arthritis study and the association between them in the selected population, are analyzed the values of triglycerides, cholesterol, HDL- Cholesterol, LDLCholesterol, that are the principal analytical components of Dyslipidemia disease, the values of glycemi that is the principal analytical component of Diabetes Mellitus disease, and the values of fibrinogen and erthrosediment that are the principal analytical components of Rheumatoid Arthritis disease. In the study are taken 289 individuals ( 154 female dhe 135 male ),that are $85.8 \%$ with Diabetes Mellitus, $9.7 \%$ with Dyslipidemia and 4. 5\% with Rheumatoid Arthrytis to a group of individuals in the Shijak population. For the study was used the statistical processing with Excel and SPSS program. Individuals are divided in age groups. First age group ( 0 - 14 years old ), second age group ( 15- 24 years old), third age group ( $25-34$ years old), fourth age group ( 35- 44 years old ), age group five ( 45- 54 years old ), age group six ( 55 - 64 years old ), age group seven ( over 65 years old ). By means of calculations is found the average value of each analytical component. For the description of statistical analyzes is used graphic construction. For changing analytical values between components and for the study of dependence between them is realized correlation method. Normal values of analytical components and analytical methods are taken from laboratory manuals ( Gesan Production 2015 ) (Human Gesellschaft ).

\section{Table 1: Normal Value of Principal Analytical Components}

\begin{tabular}{|l|l|}
\hline Components & Normal values \\
\hline Triglycerides & Male $(60-165 \mathrm{mg} / \mathrm{dl})$ \\
& Female $(40-140 \mathrm{mg} / \mathrm{dl})$ \\
\hline Cholesterol & $(180-220 \mathrm{mg} / \mathrm{dl})$ \\
\hline HDL- Cholesterol & $(>35 \mathrm{mg} / \mathrm{dl})$ \\
\hline LDL- Cholesterol & $(130-190 \mathrm{mg} / \mathrm{dl})$ \\
\hline Glycemi & $(70-100 \mathrm{mg} / \mathrm{dl})$ \\
\hline Erythrosediment & $(200-400 \mathrm{mg} / \mathrm{dl})$ \\
\hline Fibrinogen & $(<10 \mathrm{~mm} / \mathrm{h})$ \\
\hline
\end{tabular}

\section{Results and Discussions}

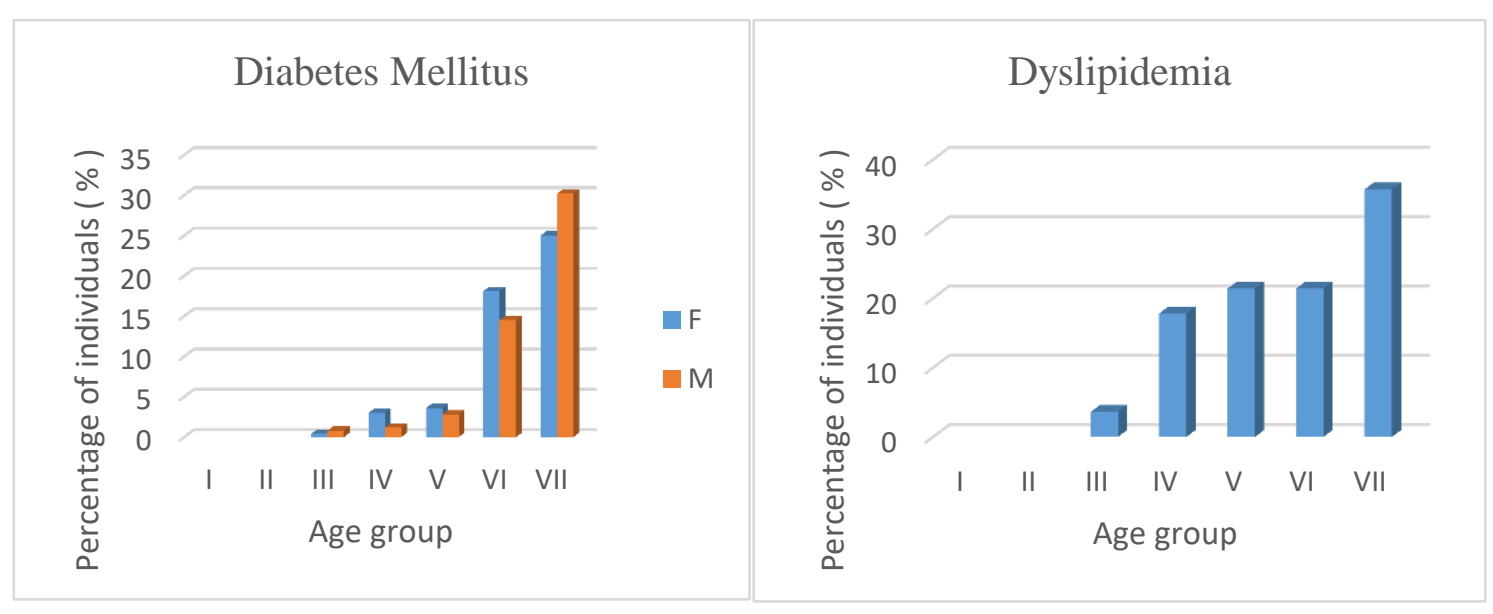

Figure 2: Diabetes Mellitus Disease

Figure 3: Dyslipidemia Disease 
Table2: Correlation Value ( with Excel program )individuals with Diabetes Mellitu

\begin{tabular}{|l|c|}
\hline Components & Correlation value \\
\hline Glycemi- Triglyceride & 0.157 \\
\hline Glycemi- Cholesterol & -0.015 \\
\hline Glycemi- HDL-Ch & -0.019 \\
\hline $\begin{array}{l}\text { Glycemi- LDL-Ch } \\
\text { Glycemi- } \\
\text { Erythrosediment }\end{array}$ & 0.083 \\
\hline \begin{tabular}{l} 
Glycemi- Fibrinogen \\
\hline
\end{tabular} & -0.205 \\
\hline
\end{tabular}

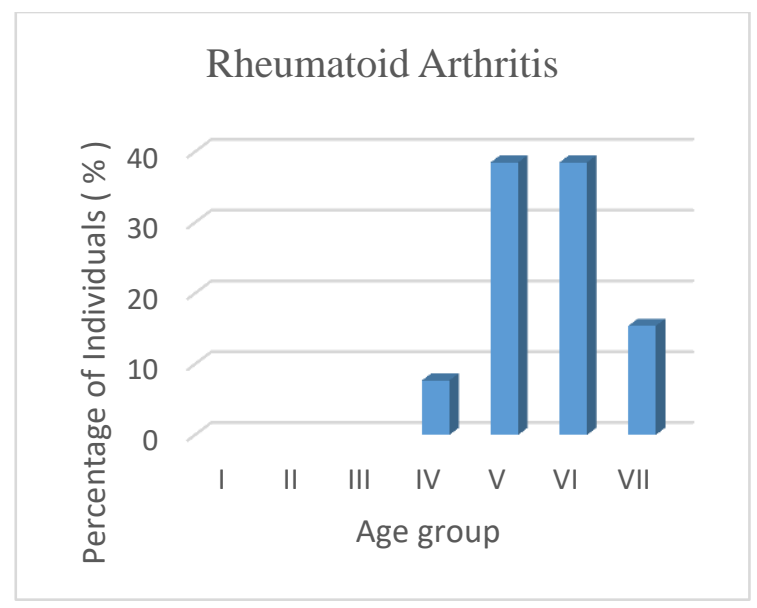

Figure 4: Rheumatoid Arthritis Disease

First and second age groups have no individuals with DM and Dyslipidemia. With age the number of individuals with DM and Dyslipidemia increases. First, second and third age group have no individuals with RA. Age group V ( 45 - 54 years old ) and VI ( 55- 64 years old ) has larger numbers of individuals with RA.

Table 2. shows the correlation values between glycemia and principal lipid components, erythrosediment and fibrinogen for individuals with DM. High correlation values are between glycemi- triglyceride with value 0.157. Individuals with Diabetes Mellitus have a high tendency to be affected by Dyslipidemia. Between glycemia- erythrosediment has low correlation value.

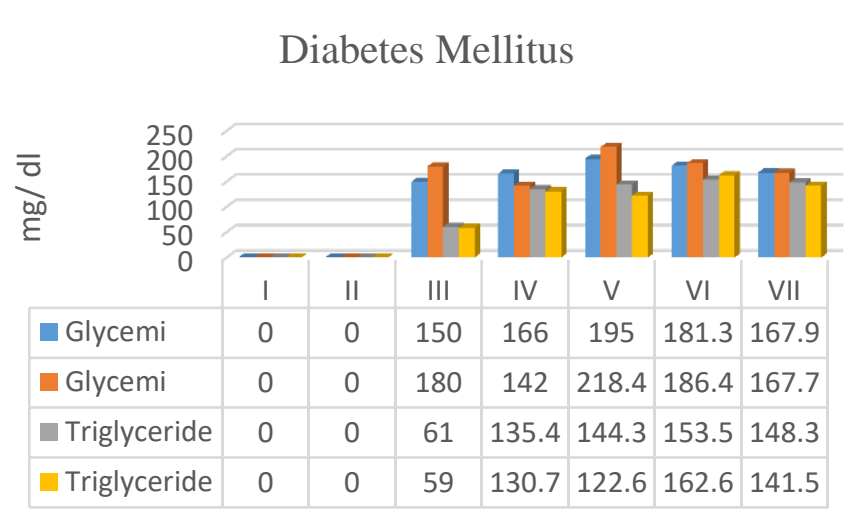

Figure 5: Average Value for Analytic Components for Each Age Group, Male and Female ( Glycemi, Triglyceride ). 
The highest average glycemic value for females and males is in V age group, respectively $195 \mathrm{mg} / \mathrm{dl}$ and $218 \mathrm{mg} / \mathrm{dl}$, higher for males. The higher average value of triglycerides for patients with Diabetes Mellitus for female and male gender is in the VI age group, respectively $153.5 \mathrm{mg} / \mathrm{dl}$ and $162 \mathrm{mg} / \mathrm{dl}$, higher value for males. Between Glycemi- Triglycerides has correlation.

Table 3: Correlation Value ( Pearson Correlation with SPSS Program )

\begin{tabular}{|l|l|l|l|l|l|l|}
\hline \multicolumn{2}{|l|}{ Correlations } & Glycemi & Triglycerides & Cholesterol & LDL & HDL \\
\hline \multirow{3}{*}{ Glycemi } & $\begin{array}{l}\text { Pearson } \\
\text { Correlation }\end{array}$ & 1 & $.157^{*}$ & -.015 & -.019 & .083 \\
\cline { 2 - 7 } & Sig. (2-tailed) & - & .025 & .823 & .854 & .432 \\
\cline { 2 - 8 } & $\mathrm{N}$ & 247 & 206 & 212 & 93 & 91 \\
\hline
\end{tabular}

Between Glycemi and Triglycerides correlation is significant at the 0.05 level.

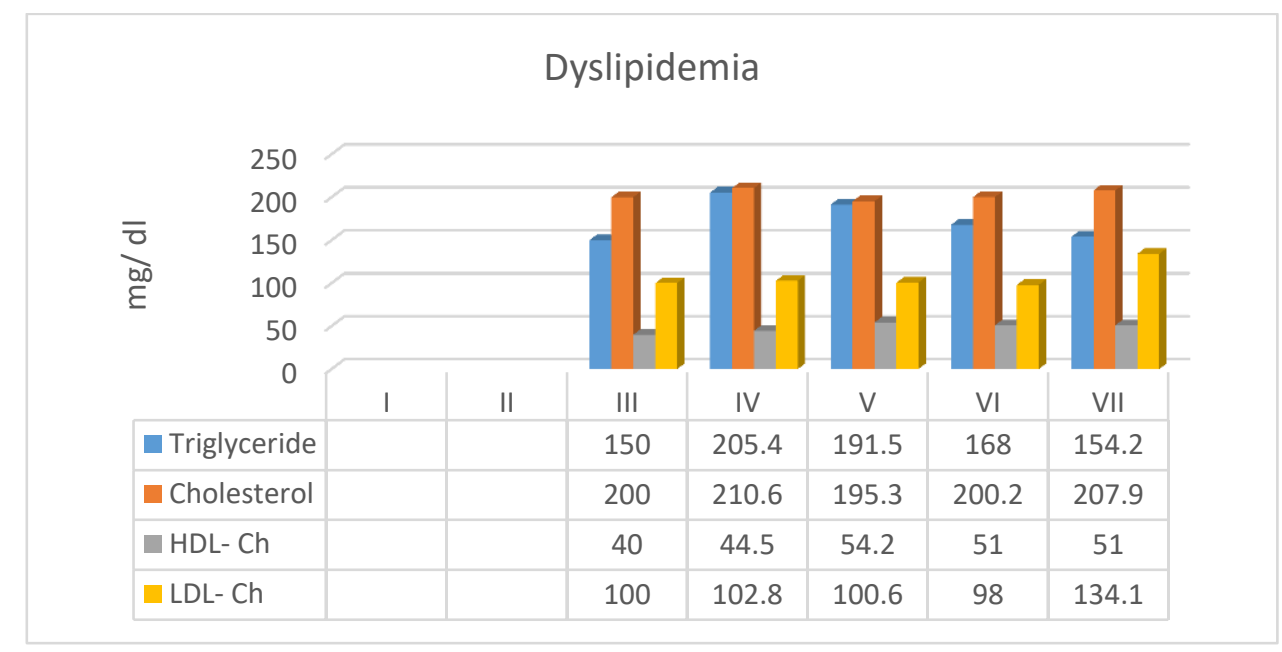

Figure 6: Average Value for Analytic Components for Each Age Group, ( Triglyceride, Cholesterol, HDL- Cholesterol, LDL- Cholesterol ).

Higher value of Triglycerides and Cholesterol in individuals with Dyslipidemia is in the IV age group, for triglycerides with value $205.4 \mathrm{mg} / \mathrm{dl}$ and for cholesterol with value $210.6 \mathrm{mg} / \mathrm{dl}$. Highest average LDLCholesterol value is in VII age group. The lowest average value of HDL- Cholesterol is in the third age group. 
Dyslipidemia

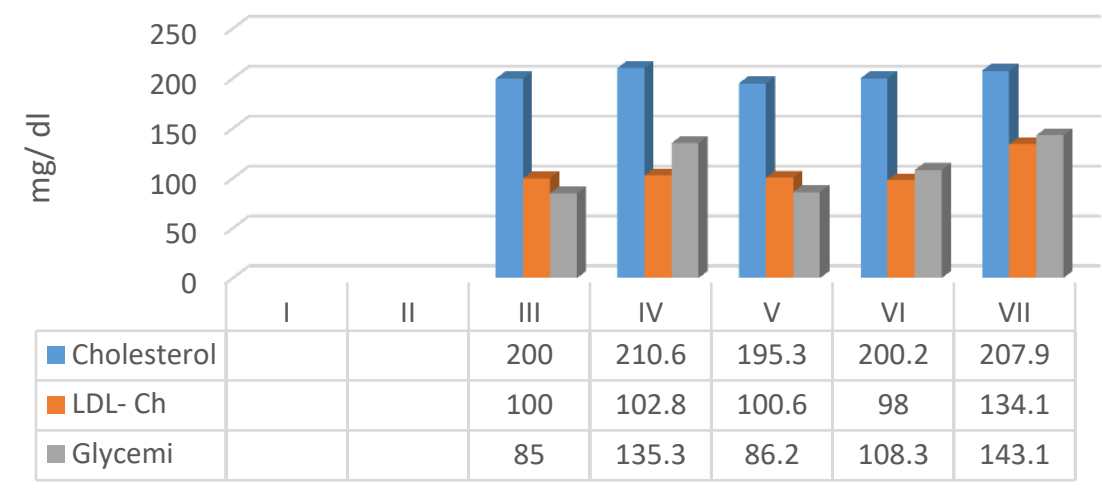

Figure 7: Average Value for Analytic Components for Each Age Group （Glycemi, LDL- Ch, Cholesterol )

The highest average glycemic value in individuals with Dyslipidemia is in VII age group, with value 143.1 $\mathrm{mg} / \mathrm{dl}$.

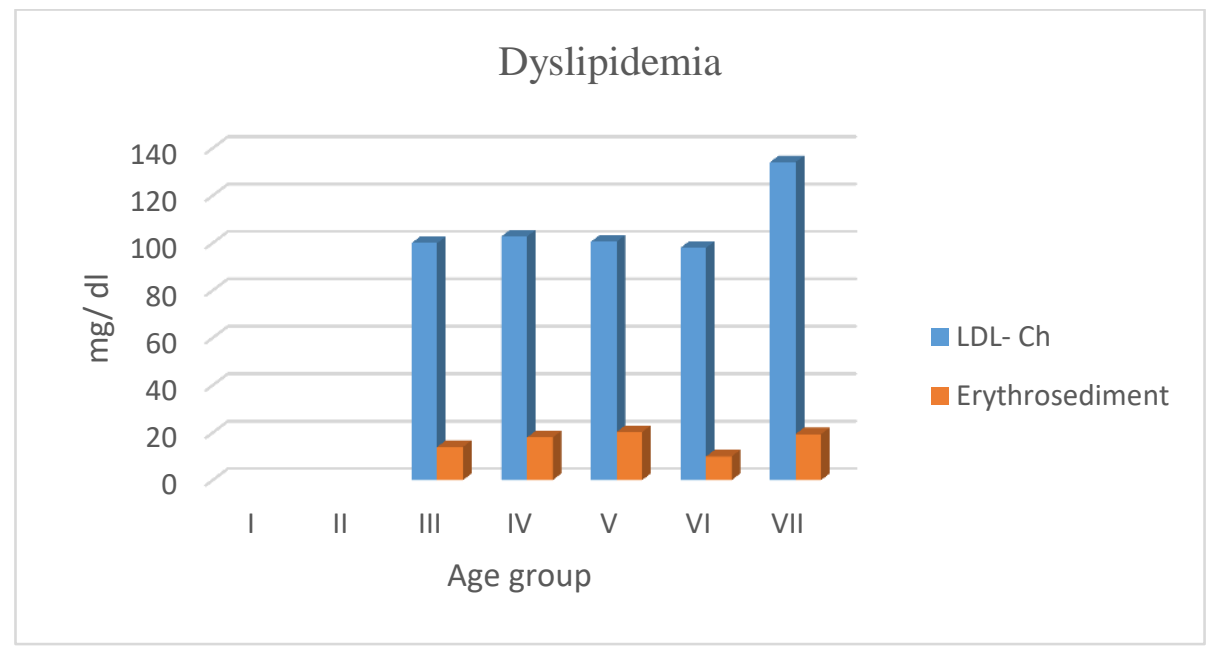

Figure 8: Average Value for Analytical Components for Each Age Group ( Erythrosediment, LDLCh)

Highest erythrosediment value is in the age group V, with value $20.3 \mathrm{mg} / \mathrm{dl}$.

Table 4: Correlation value ( with Excel Program )

\begin{tabular}{|l|l|}
\hline Compenents & Correlation value \\
\hline Triglyceride- Glycemi & 0.125 \\
\hline Cholesterol- Glycemi & 0.43 \\
\hline HDL- Ch - Glycemi & 0.086 \\
\hline LDL- Ch - Glycemi & 0.44 \\
\hline Triglyceride- Erythrosediment & 0.029 \\
\hline Cholesterol- Erythrosediment & 0.136 \\
\hline HDL-Ch- Erythrosediment & -0.59 \\
\hline LDL- Ch - Erythrosediment & 0.309 \\
\hline Triglyceride- Fibrinogen & 0.58 \\
\hline Cholesterol- Fibrinogen & -0.36 \\
\hline HDL- Ch - Fibrinogen & -0.099 \\
\hline LDL- Ch - Fibrinogen & -0.486 \\
\hline
\end{tabular}


Higher correlation values are between LDL- Ch - Glycemi ( 0.44 ), Cholesterol - Glycemi ( 0.43 ), HDL-Ch - Erythrosediment ( - 0.59 ), Triglyceride - Fibrinogen ( 0.58 ).

Table 5: Correlation Value ( Pearson Correlation with SPSS Program )

\begin{tabular}{|l|l|l|l|l|}
\hline \multicolumn{2}{|c|}{ Correlations } & $\begin{array}{l}\text { Cholest } \\
\text { erol }\end{array}$ & Triglyceride & Glycemi \\
\hline \multirow{2}{*}{ Cholesterol } & $\begin{array}{l}\text { Pearson } \\
\text { Correlation }\end{array}$ & 1 & $.451^{*}$ & $.550^{* *}$ \\
\cline { 2 - 5 } & Sig. (2-tailed) & .016 & .007 \\
\cline { 2 - 5 } & $\mathrm{N}$ & 28 & 28 & 23 \\
\hline *. Correlation is significant at the 0.05 level (2-tailed). \\
\hline **. Correlation is significant at the 0.01 level (2-tailed). \\
\hline
\end{tabular}

Between Cholesterol - Glycemi, the correlation is significant at the 0.05 level.

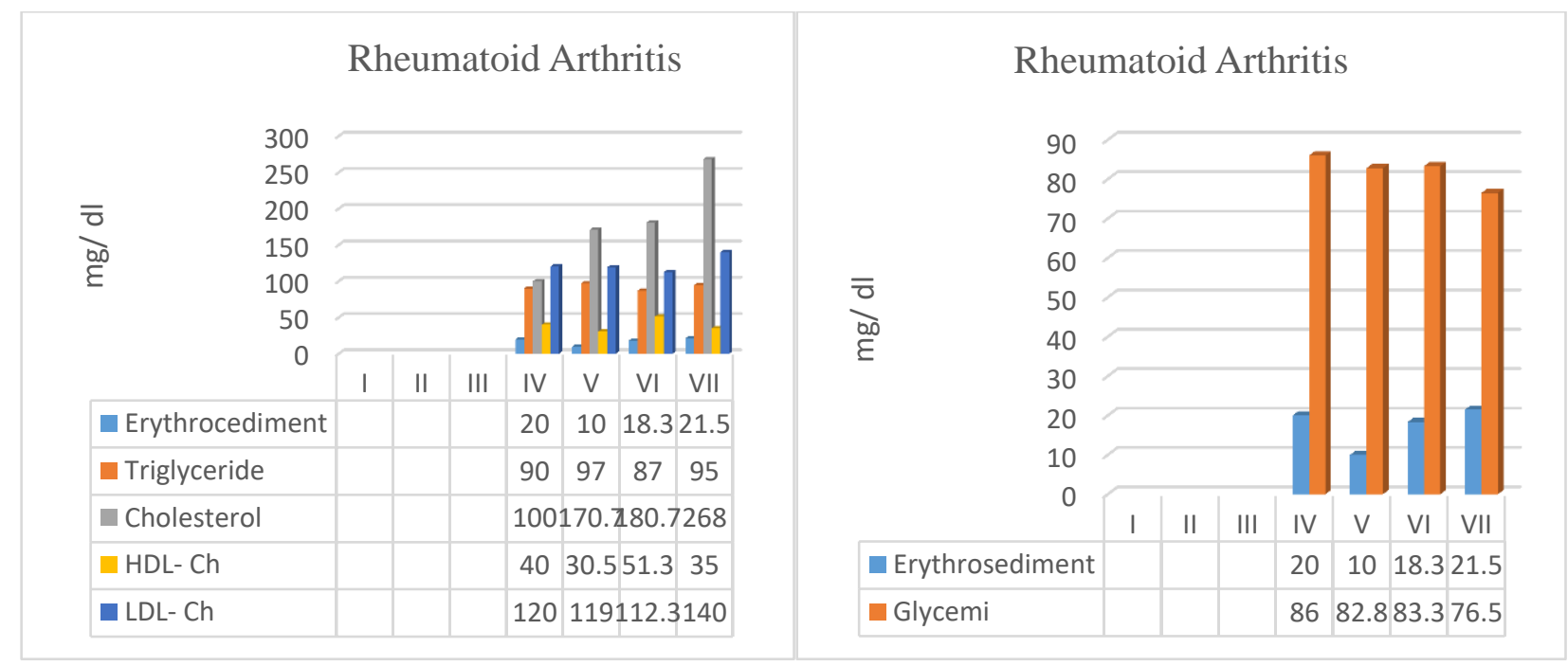

Figure 9: Average value for analytical components Figure 10: Average value for analytic components ( triglyceride, Cholesterol, HDL-Ch, LDL- Ch ) ( erythrosediment, glycemi )

For individuals with Rheumatoid Arthritis, higher Erythrosediment, Cholesterol and LDL- Ch values are in the VII age group, with values respectively $21.5 \mathrm{mg} / \mathrm{dl}, 268 \mathrm{mg} / \mathrm{dl}, 140 \mathrm{mg} / \mathrm{dl}$. Higher triglyceride values and the lowest HDL- Cholesterol values are in the V age group, with value respectively $97 \mathrm{mg} / \mathrm{dl}$ and 30.5 $\mathrm{mg} / \mathrm{dl}$. The highest average glycemic value is in the IV age group with value $86 \mathrm{mg} / \mathrm{dl}$.

The female gender is in greater numbers than the male gender in patients with Rheumatoid Arthritis.

Table 6: Correlation Value ( with Excel Program )

\begin{tabular}{|l|l|}
\hline Components & Correlation value \\
\hline Erythrosediment- Triglyceride & 0.506 \\
\hline Erythrosediment- Cholesterol & 0.216 \\
\hline Erythrosediment - HDL- Ch & 0.68 \\
\hline Erythrosediment - LDL- Ch & 0.93 \\
\hline Erythrosediment - Glycemi & -0.3 \\
\hline
\end{tabular}


For the individuals with Rheumatoid Arthritis, the higher correlation value are between ErythrosedimentLDL- Ch ( 0.93 ). Individuals with Arthritis kanë mundësi më të madhe të preken nga Dyslipidemia.

\section{Conclusion}

Age is a risk factor. With age the number of cases with Diabetes Mellitus increases. VII age group ( 65 years and olders ), has largers numbers of individuals with Diabetes Mellitus. Young age groups as a first and second age group there are no individuals with Diabetes Mellitus. The highest average glycemic value is in the age group V ( $45-54$ years old ), with average value $195 \mathrm{mg} / \mathrm{dl}$ for female gender and $218.4 \mathrm{mg} / \mathrm{dl}$ for male gender. In individuals with Diabetes Mellitus correlation value between Glycemi- Triglycerides is significant, with Pearson correlation values 0. 157. Individuals with Diabetes Mellitus have a high tendency to be affected by Dyslipidemia. Correlation value between Glycemi- Erythrosediment is low in individuals with Diabetes Mellitus.

With age the number of individuals with Dyslipidemia increases. Age group VII ( 65 years and older ) has the largest number of individuals with Dyslipidemia. The first and second age groups have no individuals with Dyslipidemia. Higher values of triglycerides and cholesterol are in the IV age group ( 35- 44 years old ). Higher LDL-cholesterol values are in the VII age group ( 65 years and older ). The lowest values of HDLcholesterol are in the IV age group ( 35- 44 years old ). Between Cholesterol- Glycemia has correlation with significant value according to the value of the Pearson correlation 0.550. Individuals with Dyslipidemia have a high chance to be affected by Diabetes Mellitus.

Correlation values between Triglycerides- Fibrinogen are 0.58 ( calculated with excel program ). Individuals with Dyslipidemia have a high tendency to be affected from Rheumatoid Arthritis.

From the study data, there is no individuals with Rheumatoid Arthritis in the age group I, II, III and the largest number of individuals with Rheumatoid Arthritis are female. The largest number of individuals with Rheumatoid Arthritis are in the V and VI age group. Higher erythrosediment values are in the VII age group. Higher correlation value in individuals with Rheumatoid Arthritis are between Erythrosediment- LDLCholesterol, which means that individuals with Rheumatoid Arthritis have a high tendency to be affected from Dyslipidemia.

\section{Importance of the Study}

The knowledge gained from this study are important to determine the correct diagnosis for primary and concomitant diseases recognizing risk factors. Prevention of diseases at an early stage and prevention of comorbidities to avoid deteriorating health. Health control and providing proper treatment from the doctor's recommendation. Implementing a regular life style, physical activity, balancing the food diet and not consuming tobacco and alcohol.

\section{References}

1. Abdalrashid Faisal M Halawani, Zubaidah Saad Alahmari, Dina Abdullah Asiri, Abdullah Ahmed Albraheem, Abdullah Munahi A Alsubaie, Abdulrahman Ghazi Alqurashi , Feher Mohammed Alturkistani, Mohammed Khamis Albalawi, Fahad Nasser Abdulrahman Alzaid, Mansour Musaad T Alsaluli , Mohammad Saleh Sahhab Alghamdi ( 2019 ). Diagnosis and Management of Dyslipidemia: 67

2. American Diabetes Association 2014. Diabetes Care: 81

3. American Family Physician ( 2011 ). Diagnosis and Management of Rheumatoid Arthritis: 1245

4. Building Healthy lifestyles, Clinical Guide ( 2006 ). Vascular Protection Dyslipidemia: 5

5. Gesan Production s. 1. r ( 2015) www.gesanproduction.it

6. Human Gesellschaft fur Biochemica und mbH Max- Planck- Ring 2165205 Wiesbaden Germany 
7. Jéssica Barbieri, Paula Caitano Fontela, Eliane Roseli Winkelmann, Carine Eloise Prestes Zimmermann, Yana Picinin Sandri, Emanelle Kerber Viera Mallet, and Matias Nunes Frizzo ( 2015 ). Anemia in Patients with Type 2 Diabetes Mellitus: 1

8. Jim Gerber. Clinical Standards, Protocols, and Education (CSPE) Committee Owen Conway, DC; Daniel DeLapp, DC, DABCO, ND, LAc; Lorraine Ginter, DC; Ronald LeFebvre, DC; Karen E. Petzing, DC; Ravid Raphael, DC, DABCO; Anita Roberts, DC; Cherye Roche DC .Dyslipidemia:5, 6

9. MOH ( Ministry of Health Singapore ) Clinical Practice Guidelines ( 2016 ). Lipids: 22

10. Ming-Chi Lu, Shih-Tang Yan, Wen-Yao Yin, Malcolm Koo, Ning-Sheng Lai ( 2014 ). Risk of Rheumatoid Arthritis in Patients with Type 2 Diabetes: A Nationwide Population-Based CaseControl Study.

11. Shelja Deswal, Mohit Deswal, Vishal Goel, Harpreet Singh ( 2015 ). Dyslipidemia in Rheumatoid Arthritis: 180, 181

12. The Journal of Rheumatology ( 2007 ). Rheumatoid Arthritis and Diabetes Mellitus: Evidence for an Association: 460

13. Yasin Simsek, Halit Diri, Fahri Bayram ( 2015 ) Tip 2 Diabetes Mellitus ve Dislipidemi.

14. Wikipedia. https://sq.wikipedia.org/wiki/Shijaku 